\title{
ANALYSIS OF ULTRASOUND GENERATION METHODS AND SYSTEMS USED IN PHYSIOTHERAPY
}

\author{
Diana Mura Badea ${ }^{1}$, Dumitru Vlad ${ }^{1}$, Valentina Daniela Bajenaru ${ }^{1}$, Simona Elena Istriteanu ${ }^{1}$ \\ ${ }^{1}$ National Institute of Research and Development in Mechatronics and Measurement Technique, \\ INCDMTM Bucharest \\ Emails: dianammura@gmail.com, didivlad2006@yahoo.com, \\ valibajenaru@yahoo.com,simona.istriteanu@incdmtm.ro
}

\begin{abstract}
The research aims to create a mechatronic system based on ultrasound, which will be used in medical recovery, especially in physiotherapy. Ultrasound therapy requires the use of waves (ultrasound) at a frequency of $1 \mathrm{MHz}$ or $3 \mathrm{MHz}$ which means an emission of these ultrasounds in two types: continuous emission or emission by pulses (interrupted). These ultrasounds have an additional component compared to most types of energy, namely it is not just a radiation energy but has a thermal component, respectively the thermal energy released will contribute by increasing the temperature in the target tissue and thus can produce a double benefit for medical recovery.
\end{abstract}

Keywords: Mechatronic System, Ultrasound, Medical Recovery, Physiotherapy (centre).

\section{Introduction}

Good health is an important concern of European citizens. The European Union (EU) pursues a higher level of health protection through its policies and activities, in accordance with Article 168 of the Treaty on the Functioning of the European Union. EU action in the field of health aims to improve public health, prevent diseases and threats to health (including those related to lifestyle), and promote research. The EU does not define health policies or organize or provide health and healthcare services, but its actions complement national policies and support cooperation between Member States in the field of public health.

Sports medicine is a branch of medicine that groups together the prevention, diagnosis and treatment of accidents and illnesses that occur during physical activity and sports, as well as advice and preventive measures to maintain and improve the physical condition of athletes of all ages and levels. Sports medicine has evolved and become a medical specialty that has brought important innovations in many medical fields.

Kinesiotherapy, also called physical therapy, kinesitherapy or medical kinetology is a type of medical gymnastics, which means, etymologically, movement therapy. It is used for medical recovery and consists, in addition to performing exercises, in a set of techniques and methods applied according to each person and the condition they present. Physical therapy is part of physical medicine and studies the neuromuscular and articular mechanisms that ensure normal motor activities in humans. It is based on the movement performed through wellstructured medical recovery programs, which aim to restore diminished functions.

Physiotherapy is the therapeutic use of natural agents such as water (thermal cures, balneotherapy), mud (mud therapy), some mineral oils such as paraffin (paraffin therapy), climate (sun, altitude), heat and electricity (low or high frequency direct or discontinuous currents, diathermy, ionization, ultrasound). Physiotherapy, a re-education technique, is indicated in all degenerative diseases of the skeleton (especially in osteoarthritis) and in inflammatory joint diseases (gout, rheumatoid arthritis, etc), generally between two seizures. It allows pain relief, prevention or reduction of joint stiffness, restoration of muscle strength around a joint.

\section{Ultrasound and its use in Technological Processes}

Sound waves can be described and identified using acoustic parameters. Frequency is the number of complete cycles performed by the sound wave in a second and is measured in hertz $(\mathrm{Hz})$. Figure 1 shows the sound vibrations depending on the frequency and fields of application.

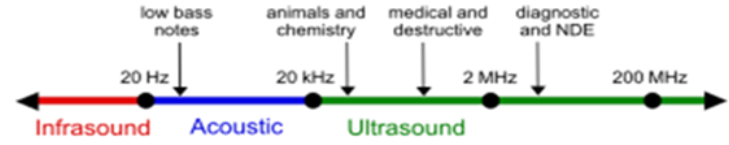

Figure 1: The Representation of sound vibrations depending on frequency and fields of application 
Depending on how the waves can be intercepted by the human ear, vibrations are divided into audible sounds (with frequencies between $20 \mathrm{~Hz}$ and 20,000 $\mathrm{Hz}$ ), infrasound (frequencies below $20 \mathrm{~Hz}$ ) and ultrasound (frequencies with values above $20 \mathrm{~Hz}$ ). $000 \mathrm{~Hz}$ ).Ultrasound is mechanical vibration with frequencies between $20 \mathrm{~Hz}-200 \mathrm{MHz}$. They usually have low intensity. High intensity ultrasound is obtained by electromechanical processes that are based on the piezoelectric phenomenon and/or the magnetostriction phenomenon.

Ultrasound (US) is a form of mechanical energy that propagates in the form of frequency waves above the perception limit of the human ear. Man perceives sounds with a frequency between 16 and $20,000 \mathrm{~Hz}$. Sounds with a frequency above the human audibility limit $(20 \mathrm{MHz})$ are called ultrasound, and those with a frequency below this are called infrasound.

If a particle in an elastic medium performs a back and forth motion toward the equilibrium position, a movement called mechanical oscillation or vibration, an energy transfer takes place in the surrounding environment. The oscillating particle interacts with the neighboring ones and thus the wave propagates from close to close. The region of space where ultrasonic waves are located (in other words, the US field) is represented by cyclic oscillations in space and time. The movement of the particles around the equilibrium position is repeated at certain time intervals.

\subsection{Ultrasound Properties}

The period ( $\mathrm{T}$ ) is the time required for a particle to describe a complete oscillation and is expressed in seconds.

Frequency (f) is the number of oscillations performed per unit time (second). The unit of frequency is Hertz (Hz). A frequency of $1 \mathrm{~Hz}$ corresponds to an oscillation per second $(\mathrm{f}=1 / \mathrm{T})$.

The amplitude of the oscillation is the absolute value of the maximum distance traveled by the particle around the equilibrium position.

The wavelength is the distance between two maxima or between two successive points in the same phase.

The speed of the ultrasound expresses the distance traveled by the US in the unit of time. It is measured in $\mathrm{m} / \mathrm{s}$. The propagation speed of the US is $331 \mathrm{~m} \mathrm{/} \mathrm{s} \mathrm{in} \mathrm{air,} 1430 \mathrm{~m} / \mathrm{s}$ in water and much higher in solid bodies, being dependent on the density and elasticity of the environment. Sound waves do not propagate in a vacuum, and in gases they propagate quite hard, due to the large distance between molecules.

Acoustic energy. The ultrasonic wave transports and transfers some of the energy to the environment, causing oscillations of particles in the environment. It is measured in Jouli (J).
Ultrasound intensity is the amount of energy that travels through the unit of surface in a unit of time. It is expressed in $\mathrm{W} / \mathrm{cm} 2$. The US intensity decreases proportionally with the distance traveled, the higher the acoustic attenuation, the higher the frequency. So as the frequency increases, the depth of penetration decreases. At one frequency, the depth of penetration of the US is limited by the decrease in intensity.

The acoustic impedance expresses the resistance to the passage of waves, being the product between the density of the environment and the US speed. The acoustic impedance is therefore a material constant: $Z=\rho \cdot c$. It is measured in rayl; 1 rayl $=1 \mathrm{Kg}$ - $\mathrm{m}^{-2} \cdot \mathrm{s}^{-1}$. The separation limit between two environments with different density, so with different acoustic impedance, is called the interface. At the interface level, the ultrasonic pulse is: reflected, refracted, dispersed, absorbed or attenuated.

The reflection consists in returning to the initial environment a part of the US beam when crossing an interface, depending on the acoustic impedance of the two environments. The direction of the reflected beam depends on the angle of the incident beam with the interface.

Dispersion consists in reirradiation, ie in the emission of new spherical waves in areas with different acoustic impedances and with dimensions smaller than the wavelength. The dispersal leads to an increase in the US's range.

Attenuation occurs through: absorption, dispersion, reflection. The attenuation is directly proportional to the square of the distance traveled and to the frequency of the US beam. High frequency waves are attenuated after a short distance, and low frequency waves penetrate deep.

Diffraction. When the US beam passes at a distance of less than one or two wavelengths from an obstacle, the direction of wave propagation is deflected behind it. Behind the obstacle there are areas of acoustic shadow, and in front of it there is interference of waves, as a result of the action of several waves on the same particles. If the waves are in the same phase, the effect accumulates and we are dealing with a constructive interference, and if they are in the antiphase the effect is canceled, the interference being destructive.

Acoustic power is the amount of energy that travels through an area in a unit of time and is measured in watts.

\section{Ultrasound Interactions}

The possible interaction mechanisms when ultrasonic waves pass through an environment are the following:

- thermal mechanism

- cavity

- tensioning mechanism. 


\subsection{The Thermal Mechanism}

The interaction of ultrasound with matter is followed by the absorption by the environment of a part of the beam energy, which is transformed into heat. The heat generated per unit volume of the medium is proportional to the acoustic intensity and the absorption coefficient and inversely proportional to the density of the medium and its specific heat.

Taking into account the effect of thermal conduction, it is estimated that after a while the temperature will reach an equilibrium value. For spherical bodies the increase in equilibrium temperature, as well as the time to reach this temperature are proportional to the square of the radius.

\subsection{Cavitation}

Cavitation can be defined as the dynamic phenomenon of appearance, development and disappearance by implosion of gas bubbles (cavities), in the mass of a moving liquid. The appearance of these bubbles occurs when the pressure drops below a critical value, represented by the vaporization pressure.

In a narrow sense of the word, cavitation means the dynamic process of forming and collapsing cavities in a stream of liquid, which contains vapors and gases. In normal liquids, these cavities are formed when the pressure at certain points is reduced to the value of the vaporization pressure of the liquid. In these points or areas, the liquid boils and vapor bubbles are formed, which together with the liquid reach the area of high pressures, where the collapse of these cavities takes place. Condensation of vapor bubbles in this region causes local hydraulic shocks or overpressures, when, at the end of condensation, the surrounding liquid particles advance towards the center of the bubble, collide and stop abruptly. In this place the kinetic energy is transformed into elastic deformation energy. In areas where the cavitation process ends, the increase in pressure due to hydraulic shocks can reach values of tens, hundreds, or thousands of atmospheres, and the energy of these shocks propagates in the form of pressure waves that manifest outside through strong vibrations and characteristic noises. A foamy environment appears in the vaporization area, which can be observed with the naked eye through a transparent pipe.

Cavitation can be stable or temporary. The diameter of a stable cavity that can resonate to produce the phenomenon of cavitation in water, when passing ultrasonic waves with a frequency of 1 $\mathrm{MHz}$, is about $3.5 \mu \mathrm{m}$. The acoustic intensity required for the cavitation to appear in water is $\approx 30$ $\mathrm{mW} / \mathrm{cm}^{2}$. When the bubble expands and contracts, microcurrents are formed in which the speed gradients can be very high.
The temporary cavitation is more violent than the stable one. When a gas bubble in an environment is subjected to the action of the ultrasonic field, with a high pressure amplitude, it increases its radius several times from the initial value and then explodes violently. In the final stages of the explosion, the kinetic energy is given to an extremely small volume and for this reason high pressures and temperatures occur.

Temperatures of $3500 \mathrm{~K}$ and pressures of 104 atm were estimated at the temporary cavitation. Under these conditions, the environment suffers, at least due to mechanical shock waves and high temperatures. Temporary cavitation occurs especially when the intensity exceeds a threshold value, depending on the experimental conditions:

- for continuous ultrasonic waves, the higher the ultrasound frequency, the higher the ultrasonic threshold intensity will have to be to produce the cavity;

- the increase of the ambient pressure leads to the increase of the ultrasonic threshold intensity for the cavity production;

- the increase of the temperature decreases the ultrasonic threshold intensity;

- increasing the volume of the liquid exposed to ultrasound decreases the ultrasonic threshold intensity.

\subsection{The Tensioning Mechanism}

In heterogeneous systems subjected to the action of an ultrasonic field, the resulting voltages or forces appear, classified as follows:

- oscillating forces, whose average in time is equal to zero and which produce a pressure on the bodies with different density from the environment;

- displacement forces, which have a time average other than zero and can cause the displacement of inhomogeneities in the environment with different speeds;

- forces due to the variation of viscosity during the application of ultrasound.

The three forces presented determine the appearance in the ultrasonic field of some microcurrents, highlighted near the vibrating gas bubbles.

\section{Ultrasound Production}

Ultrasonic waves are obtained by mechanical, magnetostrictive and piezoelectric methods. The body that vibrates and generates ultrasonic waves is called the acoustic source or the ultrasound source. The basis for obtaining ultrasound is most often the piezoelectric phenomenon, an effect discovered in 1880 by Pierre and Jacques Curie. The appearance of electrical polarization on the surface of a crystal when a mechanical pressure or traction is exerted on it is called a direct piezoelectric effect. Applying an 
electric field on the surface of a piezoelectric crystal leads to its contraction or expansion and the emission of acoustic waves. This phenomenon is called the reverse piezoelectric effect.

The most used piezoelectric materials are: barium titanate, lead zirconate (piezoceramic materials) and polyvinylidene fluoride (plastic). Natural or synthetic quartz also has piezoelectric properties, its advantages being mechanical strength and low internal friction. Piezoceramic materials have a better efficiency of conversion of electrical energy into mechanical energy, are cheap, easy to process and require low voltages.

The magnetostrictive effect consists in the fact that some ferromagnetic materials change their dimensions when magnetized. When these materials are in a variable magnetic field, they begin to oscillate, becoming sources of acoustic waves.

In the both cases of ultrasound generation, it is necessary that the dimensions of the oscillating plates be chosen so that their own frequency coincides with the excitation frequency (frequency of the electric field, respectively of the magnetic field) so that the ultrasonic generators work in resonance mode.

Ultrasonic transducers ensure the reciprocal and successive conversion of electrical energy into mechanical energy. Their active element consists of the piezoelectric crystal. It has the shape of a disc and is covered on both sides with two layers of metal, good conductors of electricity, on which two electrodes are applied, one on each surface. Applying an electrical voltage between the electrodes will cause the crystal to deform and consequently the emission of mechanical energy to both surfaces. The metal layers have both the role of transferring the electrical voltage to the crystal, and that of taking over the electrical impulse created at its surface after the action of ultrasound reflected in the tissues. This created electrical impulse is then led to the amplification system of the device. The thickness of the piezoelectric disk determines the nominal frequency. An acoustic lens, with a thickness equal to a quarter of the wavelength of the electrical excitation frequency, is sometimes arranged on the inner surface from which the medium is acting.

The lens is also called an adaptive layer of quarter wavelength, its role being to focus and make each electrical impulse to strengthen the other, thus increasing the efficiency of the transducer. An insulating layer with an impedance similar to that of the body is placed in front of the lens. A layer of USabsorbing material is then inserted behind the piezoelectric disk and then to dampen vibrations that do not have the desired frequency. This whole assembly is surrounded by an acoustic insulating layer and is inserted into a plastic cover with which the operator comes into contact during the examination.
The back face of the piezoelectric material is lined with an attenuating material, having the role of reducing the sound resonance capacity.

Ultrasound beam. The piezoelectric material does not emit a single ultrasonic wave, but a beam that starts from the entire surface of the material. In a first portion, of a few $\mathrm{cm}$, this beam is narrow and has a cylindrical shape, the waves in the component having practically a parallel arrangement. This nearby area is called the Fresnel area. Another portion follows, called the remote area or the Fraunhofer area, in which the waves become divergent and the beam has the shape of a cone trunk. The length of the Fresnel zone and the divergence of the Fraunhofer zone depend on the dimensions of the piezoelectric disk, but also on the frequency of the ultrasounds produced by it. Increasing the frequency of the ultrasound or the diameter of the piezoelectric disk causes the Fresnel area to increase and the divergence angle to decrease.

\section{Applications of Ultrasound in Technological Processes}

Ultrasound is usable in all stages of a technological process, from raw material conditioning to process control.

Examples of technological operations performed under the action of ultrasound:

- dispersion, the physical process of spreading the particles of a substance among those of another substance;

- cleaning, based on the cavitation phenomenon; ultrasonic cleaning is widely used due to the quality of the operation performed, the short working time, the diversity of materials that can be subjected to this operation;

- sedimentation, based on the agglomeration of fine particles, solid or liquid, in the area of the nodes of a stationary field produced by the propagation of ultrasound;

- filtration, the operation of separating a solid substance from a liquid;

- emulsification, based on the dispersion of particles of one liquid in another in which it is miscible, or of a solid substance in a liquid in which it does not dissolve;

- extraction, the operation of separating one or more substances from a mixture;

- stimulation of chemical reactions (e.g. polymerization ones);

- drying, the process of removing water from a material;

- crystallization, based on the difference in solubility of the components of a mixture;

- sterilization, based on the destructive action of ultrasound on microorganisms (e.g. in the food industry); etc. 


\section{Ultrasound Therapy}

Ultrasound therapy or ultrasonotherapy is a largescale therapeutic method among the measures within physiotherapy, to which can be added electrotherapy (or therapy with diadynamic currents) or other measures specific to physiotherapy.

This measure requires the use of waves (ultrasound) at a frequency of $1 \mathrm{MHz}$ or $3 \mathrm{MHz}$ which means an emission of these ultrasounds in two types: continuous emission or emission by pulses (so interrupted) of them.

These ultrasounds also have a component that most types of energy is not just a radiation energy but has a thermal component, ie the thermal energy released will contribute by increasing the temperature in the target tissue and thus can produce a double benefit.

This therapy thus has a series of components with adjacent effects in providing the entire therapeutic complex which means ultrasound treatment. Here we will talk about the thermal effect (hyperemesis), an analgesic or analgesic effect, as well as a metabolic effect but by the fact that these where they have a degree of reflection from and at the bone level will have a component in the therapy of fractures or bone cracks as well as the reflection from the muscle fibers, so the muscular or musculoskeletal component can be added (ie myorelaxation and their spasmodic decrease.

\subsection{Components of Ultrasound Physiotherapy}

- The analgesic effect is the main effect expected by the patient when he wants to resort to this therapeutic measure, because it is the symptom most noticed by the individual, but when the doctor recommends this mode of therapy he has in mind a number of other expected effects.

The analgesic effect is in fact a consequence of all the other cumulative components of ultrasound and due to the fact that the other components are effective then analgesia will also occur.

Directly speaking, analgesia occurs through the thermal energy released with ultrasound, and this thermal energy will be absorbed by the lymph nodes and nerve endings that will no longer transmit the sensation of pain.

- The metabolic effect is perhaps the broadest component of all the other effects of ultrasound therapy, namely that ultrasound will trigger a large release of mast cells, which these mast cells will produce excess histamines.

These histamines are mainly used in allergic reactions to fade the aggressive effect on the antigen and streamline the action against the antigen. The histamines will trigger bronchodilation and once this bronchodilation is produced, cellular respiration will increase which will streamline intracellular metabolic activity and roughly speaking will rehabilitate the cell.

Also in intracellular metabolism, it is exclusively based on ATP, but the nutritional component from which ATP is extracted is the carbohydrate component, and if we talk about this intracellular carbohydrate metabolism, ultrasound will activate some proteins as glycolytic ferments and will segment carbohydrate macromolecules in smaller molecules.

The metabolism also includes the activation of a series of oxidative processes and will produce reducing effects.

The main composition of collagen tissue or connective tissue is found in vessels where vasodilation will occur.

This vasodilation is due to the thermal component and thus through this vasodilation a better tissue trophy will be produced (a nourishment of the tissue) and ensuring the adequate nutrients the recovery of the tissue at the initial stage is much better.

- Myorelaxant effect means the reduction of muscle spasms. In the case of muscle pain, they are also caused by a constant additional tension in the skeletal muscles that is kept under pressure and in which there is a series of constant involuntary (spasmodic) contractures that cause pain.

There is a possible correlation of a drug therapy such as myorelaxants or antispasmodics which actually means the same effect but on a biochemical component, and ultrasound is the biophysical component, the correlation of the two: biochemical with biophysical will ensure an even more effective effect in a duration even shorter than each separately.

\section{- Bone recovery effect}

In fractures, bone cracks or other osteo-articular pathologies such as synovitis, osteo-tendinitis. In the case of bone tissue it has a specific reaction to these ultrasounds because at low doses of ultrasound, it will form a series of young bone cells with a modeling role, called osteophytes and which is the reaction of increased bone warming.

The bone can heat up due to the reflection and reflection in the bone of ultrasound, it will heat up much more than other tissues (including muscle). These osteophytes will speed up bone recovery and ensure proper bone modeling.

In the case of bone recovery, it can be supplemented by adjuvants with pharmaceuticals, such as vitamin $\mathrm{D}, \mathrm{K}$ and calcium to which must be added and the presence of sunlight that helps fix vitamins and calcium but to which is added this biophysical effect through therapy. Ultrasound means an effective combination of effects that will speed up bone recovery.

Ultrasound indications are multiple and can be given by a recovery doctor (especially), 
rheumatologist or orthopedist, but they can be performed at the doctor's indication and by a physiotherapist or a nurse in the physiotherapy department.

\section{- Orthopedic - post-traumatic indications}

Here we start from bone fractures, bone cracks but also smaller pathologies such as dislocations. This indication is most often given by an orthopedic doctor and will ensure the formation of bone callus, bone recovery through callus.

But this therapy must include a longer period of time, so that in case of bone fractures in the arm it will require a period of 6 weeks and if in the classic orthopedic recovery therapy of 12 weeks it will be reduced by half.

In leg fractures, the necessary therapy is during 12 weeks (approx. 3 months) compared to 4 and a half months during the classic therapy (18 weeks).

If we talk about the ankle fracture, it will last 5-6 weeks (as in the arm) but it means a reduction of the therapeutic duration from 10 weeks, which represents the duration in the classical therapy without ultrasound adjuvant in case of bone fracture. the ankle.

- Rheumatic indications are prescribed by a rheumatologist and means an indication for chronic conditions such as polyarthritis (ie affecting several joints simultaneously), arthritis itself means damage to the joints.

Also in rheumatic pathologies is added spondylarthropathy, ie a damage to the spondylous tissue.

In the case of abarticular rheumatism, the indication for ultrasound therapy can be made by the rheumatologist in case of diseases such as: myositis (muscle inflammation), myogelosis nodules, periarthritis or tendonitis (tendon inflammation).

The prerogative of the therapies imposed by the rheumatologist also includes chronic degenerative rheumatic pathologies: osteoarthritis, spondylosis where the main therapeutic indication is precisely this ultrasound therapy because regardless of the duration of recovery, the recovery itself by this method is very effective.

\section{- Neurological indications:}

The indications of the neurologist refer to those pathologies related to neuralgias and neuritis that mean truncated and radicular or neuronal or nervous sequelae or in case of a dysfunction on the neuromuscular plate, ie a muscular dystrophy, dystrophic muscular syndromes with spastic syndromes and hypertonic pyramidal cause and extrapyramidal.

Neuro-dermatological indications that mean the boundary between these two major categories of pathologies, ie sclerodermitis which means a pathology of neuronal or nerve transmission and a skin component ie scleroderma where the effectiveness of treatment varies from patient to patient if we talk about treatment only by ultrasound therapy and not a drug supplement.

\subsection{Contraindications}

There are also health situations in which this ultrasound therapy has relative or total contraindications, namely:

- Those skin pathologies that give increased sensitivity to any stimulus, which will be perceived as a painful stimulus and here we can talk about all dermatological or systemic pathologies of infectious nature where the general condition of the patient is altered, pain tolerance is low and where it Ultrasound therapy can cause painful sensations instead of relieving or treating them.

- Tumors of either benign or malignant nature have an absolute contraindication because these metabolic benefits that ultrasound therapy can bring will then be absorbed and spread on the tumor tissue which will have a more pronounced growth and development, the effect being therefore the opposite of the desired one.

- Cardiovascular pathologies and especially those caused by rhythm disorders, depolarization or repolarization where these cardiac and cardioelectrical disorders will be accentuated by ultrasound therapy and where there is a possibility that these pathologies may be aggravated.

- Vascular coagulation disorders or deep vein thrombosis. Through this generalized ultrasound therapy there is a risk of a thrombus leaving the thrombus due to the vasodilation effect produced and by increasing the elasticity of the vessel wall caused by ultrasound, so that this embolus can reach the circulation and cause ischemia or heart attack.

- Pregnancy, the effects of ultrasound on the fetus are minimal or minor, considering that the ultrasound itself has an ultrasound component, however at a different frequency from therapeutic ultrasound. Although there is no contraindication to ultrasound therapy in pregnancy, before starting any such therapy it is good to consult the obstetrician who is following the pregnancy to determine whether or not there is a real risk to the pregnancy.

\section{Ultrasonic Mechatronic System}

The research carried out aims to achieve by INCDMTM a mechatronic ultrasound system that will be used in physiotherapy that will have the following characteristics:

-Three levels of intensity

-Timer (5-20 min)

-Operation with electricity

-Maximum ultrasound output power: 3 watts $/ \mathrm{cm}^{2}$

-Operating frequency: $1 \mathrm{mhz}+/-5 \%$

-Battery indicator. 


\section{Technical data of the device:}

Acoustic frequency: $1 \mathrm{MHz} \pm 10 \%, \pm 3 \mathrm{MHz} 10 \%$

Output power:

$-0.5 \mathrm{~W}-10.0 \mathrm{~W}$, when the load factor $\geq 80 \%$ for $5 \mathrm{~cm}^{2}$

$-0.5 \mathrm{~W}-15.0 \mathrm{~W}$, when the load factor $\leq 70 \%$ for $5 \mathrm{~cm}^{2}$

$-0.1 \mathrm{~W}-3.0 \mathrm{~W}$, when the load factor $\leq 80 \%$ for $1 \mathrm{~cm}^{2}$

$-0.1 \mathrm{~W}-3.0 \mathrm{~W}$, when the load factor $\leq 70 \%$ for $1 \mathrm{~cm}^{2}$

Effective intensity (max.):

$-3.0 \mathrm{~W} / \mathrm{cm}^{2} \pm 20 \%(1 \mathrm{MHz})$

$-3.0 \mathrm{~W} / \mathrm{cm}^{2} \pm 20 \%(3 \mathrm{MHz})$

Environmental conditions for use:

Ambient temperature: $-10 \sim 40{ }^{\circ} \mathrm{C}$

Relative humidity: 30\% - 85\%

Atmospheric pressure: 800-1060 hPa.

\section{Conclusions}

Ultrasound is mechanical vibration with frequencies between $20 \mathrm{kHz}-200 \mathrm{MHz}$, which usually have low intensity. High intensity ultrasound is obtained by electromechanical processes that are based on the piezoelectric phenomenon and / or the magnetostriction phenomenon. Ultrasound (US) is a form of mechanical energy that propagates in the form of frequency waves above the perception limit of the human ear. Man perceives sounds with a frequency between 16 and 20,000 Hz. Sounds with a frequency above the human audibility limit (20 MHz) are called ultrasound, and those with a frequency below this are called infrasound.

The possible interaction mechanisms when ultrasonic waves pass through an environment are: the thermal mechanism, the cavitation and the tensioning mechanism.

Ultrasonic waves are obtained by mechanical, magnetostrictive and piezoelectric methods. The body that vibrates and generates ultrasonic waves is called the acoustic source or the ultrasound source.

Ultrasound is usable in all stages of a technological process, from raw material conditioning to process control. Some examples such as: dispersion, cleaning, based on the cavitation phenomenon, sedimentation, filtration, emulsification, extraction, the operation of separating one or more substances from a mixture; stimulation of chemical reactions; drying, the process of removing water from a material; crystallization, based on the difference in solubility of the components of a mixture; sterilization, based on the destructive action of ultrasound on microorganisms (eg in the food industry).

In medicine, ultrasound therapy or ultrasound therapy is a large-scale therapeutic method among measures in physiotherapy, to which can be added electrotherapy (or therapy with diadynamic currents) or other measures specific to physiotherapy. This measure requires the use of waves (ultrasound) at a frequency of $1 \mathrm{MHz}$ or 3 $\mathrm{MHz}$ which means an emission of these ultrasounds in two types: continuous emission or emission by pulses, interrupted, of them.

The research carried out aims at the realization by INCDMTM of an ultrasound device that will be used in physiotherapy that will have the following main characteristics:

-Three levels of intensity

-Operation with electricity

-Maximum ultrasound output power: 3 watts $/ \mathrm{cm}^{2}$

-Operating frequency: $1 \mathrm{mhz}+/-5 \%$

Technical data of the device:

Acoustic frequency: $1 \mathrm{MHz} \pm 10 \%, \pm 3 \mathrm{MHz} 10 \%$

Output power:

$-0.5 \mathrm{~W}-10.0 \mathrm{~W}$, when the load factor $\geq 80 \%$ for $5 \mathrm{~cm} 2$

$-0.5 \mathrm{~W}-15.0 \mathrm{~W}$, when the load factor $\leq 70 \%$ for $5 \mathrm{~cm} 2$

$-0.1 \mathrm{~W}-3.0 \mathrm{~W}$, when the load factor $\leq 80 \%$ for $1 \mathrm{~cm} 2$

$-0.1 \mathrm{~W}-3.0 \mathrm{~W}$, when the load factor $\leq 70 \%$ for $1 \mathrm{~cm} 2$

Effective intensity (max.):

$-3.0 \mathrm{~W} / \mathrm{cm} 2 \pm 20 \%(1 \mathrm{MHz})$

$-3.0 \mathrm{~W} / \mathrm{cm} 2 \pm 20 \%$ (3 MHz).

\section{References}

[1] Costantino C, Vulpiani MC, Romiti D, Vetrano M, Saraceni VM. "Cryoultrasound therapy in the treatment of chronic plantar fasciitis with heel spurs. A randomized controlled clinical study", Eur J Phys Rehabil Med. 2014 Feb;50(1):39-47. Epub 2013 Oct 30. PMID: 24172641;

[2] Dogaru, Gabriela, "Cellular research on the treatment of pulsating short waves" (in romanian) Editura Balneara, Bucharest, 2015, ISBN 978-606-8705-00-2;

[3] Lorelei Waring, Alison Hall, Sara Riley, "Musculoskeletal Ultrasound", 1st Edition Series: "How, Why and When", Elsevier, 2021, ISBN: 9780702081989;

[4] Sidney K Edelman "Understanding Ultrasound Physics" 4th ed. Edition, 2012, Editura: E.S.P. Ultrasound, ISBN-13: 978-0962644450;

[5] Soler-López, F., "Application of ultrasound in medicine, part II: The ultrasonic transducer and its associated electronics", TECCIENCIA, Vol. 8 No. 15., 14-26, Jun 2013, DOI: http:/dx.doi.org/10.18180/tecciencia.2013.15.2

[6] ULTRASONOGRAFIA; http://ultrasonografia.ro/ multimedia platform.

[7] Vivien Gibbs, David Cole, Antonio Sassano "Ultrasound Physics and Technology", 1st Edition Series: "How, Why and When", ELSEVIER, 2009, ISBN: 9780702030413;

[8] Watson, Tim, "Ultrasound in contemporary physiotherapy practice", Ultrasonics, Volume 48, Issue 4, 2008, Pages 321-329, ISSN 0041-624X, https://doi.org/10.1016/j.ultras.2008.02.004;

[9] Watson, Tim, "Ultrasound Dose Calculations", 2017, on-line, available at https://www.electrofitness.com/media/publicati ons/ultrasound-dose-calculations.pdf 